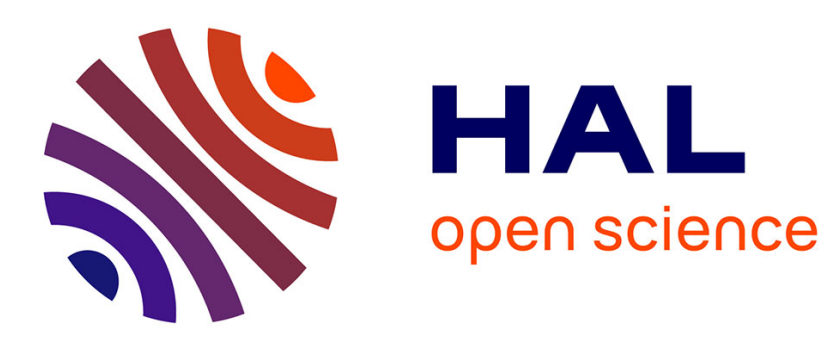

\title{
Tracking the Origin and Evolution of Plant Metabolites
}

Christophe Duplais, Nicolas Papon, Vincent Courdavault

\section{To cite this version:}

Christophe Duplais, Nicolas Papon, Vincent Courdavault. Tracking the Origin and Evolution of Plant Metabolites. Trends in Plant Science, 2020, 25 (12), pp.1182-1184. 10.1016/j.tplants.2020.08.010 . hal-02942845

\section{HAL Id: hal-02942845 \\ https://univ-angers.hal.science/hal-02942845}

Submitted on 13 Nov 2020

HAL is a multi-disciplinary open access archive for the deposit and dissemination of scientific research documents, whether they are published or not. The documents may come from teaching and research institutions in France or abroad, or from public or private research centers.
L'archive ouverte pluridisciplinaire HAL, est destinée au dépôt et à la diffusion de documents scientifiques de niveau recherche, publiés ou non, émanant des établissements d'enseignement et de recherche français ou étrangers, des laboratoires publics ou privés. 


\title{
Spotlight
}

\section{Tracking the origin and evolution of plant metabolites}

\author{
Christophe Duplais ${ }^{1}$, Nicolas Papon ${ }^{2,3}$, Vincent Courdavault ${ }^{4, *}$ \\ CNRS, UMR8172 EcoFoG, AgroParisTech, CIRAD, INRA, Université des Antilles, Université de Guyane, Cayenne, France. \\ 2 Host-Pathogen Interaction Study Group (GEIHP, EA 3142), UNIV Angers, UNIV Brest, Angers, France. \\ ${ }^{3}$ Federative Structure of Research « Cellular Interactions and Therapeutic Applications », SFR 4208 ICAT, Univ Angers, Angers, France. \\ ${ }^{4}$ Université de Tours, BBV EA 2106, Tours, France. \\ * Correspondance: vincent.courdavault@univ-tours.fr (V. Courdavault)
}

Abstract (50 words)

Iridoids are monoterpenes produced by various plants and used as chemical defenses. Lichman et al. described a timeline of molecular events that underpin the re-emergence of iridoid biosynthesis in an independent lineage of aromatic plants (catnip). This study represents a benchmark to study enzyme and metabolite evolution across lineages from the tree of life.

Keywords: Plant metabolites - Lamiaceae - iridoids - nepetalactone - enzyme evolution

\section{Core text $(1,200$ words)}

Understanding the origin and evolution of plant metabolites is fundamental to explain the distribution of natural products among plant families. These metabolites are produced by plants as a response to abiotic and biotic stress, to ensure inter- and intra-species communication and are also widely used by humans for medicine and agriculture. Mapping the presence and absence of plant metabolites across angiosperms has been undertaken for many years (1), but studying the molecular mechanisms and evolutionary processes of these metabolites still remains more challenging. To test whether a specific chemical family has evolved independently in different lineages or arisen from an ancestral pathway, the identification of genes and proteins involved in the biosynthesis of these natural products is an essential prerequisite. For example, in the biosynthesis of plant tropane alkaloids the enzymes responsible for the tropinone-reduction step are tough to have risen independently across angiosperm species (2). Conversely the norcoclaurine synthase in benzylisoquinoline alkaloid biosynthesis in angiosperms is suggested to be a monophyletic evolution prior to the emergence of eudicots (3). Deciphering the evolution of terpene synthases across plant lineages is challenging since terpenes are present in the oldest lineages of land plants that colonized terrestrial habitats (480-430 Mya) (4). Over time, terpene synthases have generated high terpenoid chemical diversity, which has played a major role in plant diversification and adaptation. In this respect, an impressive new study led by Lichman, Buell and O'Connor has shed new light on the evolution of a prominent class of monoterpenes, namely iridoids, in the well-known aromatic plants family (Lamiaceae) (5). This investigation is a tour de force 
revealing the molecular mechanisms for the loss and re-emergence of iridoid biosynthesis in the Nepeta lineage.

Iridoids are produced by several plant families and act as chemical defense against herbivores and plant pathogens. In the Lamiaceae (approximatively 200 genera, 7,000 species) which is composed of seven major subfamilies, iridoids are widely distributed across all the subfamilies but are absent in a single subfamily, i.e. the Nepetoideae (Fig. 1A). Importantly, since iridoids are also present in the sister family of the Lamiaceae, i.e. the Verbenaceae, it is likely that genes involved in iridoid biosynthesis have evolved from a common ancestor while the capacity of producing iridoids has been lost in the clade of Nepetoideae. However, there is a noteworthy exception because iridoids are found in the Nepetoideae genus Nepeta. These plants are known as catmint or catnip, due to the euphoriainducing effect of the nepetalactone iridoids on the behavior of felines. The intriguing presence of iridoids in Nepetoideae thus raises the question are the same enzymes utilized or are novel enzymes involved that have led to the re-emergence of the biosynthesis of these metabolites (Fig. 1A).

The structural core of iridoids is a cis- or trans-fused cyclopentanopyran. Unlike the biosynthesis of terpenoids generally resulting from the cyclization of a linear terpene carbocation by terpene synthase (TS), the key step to form the iridoid bicycle is a reductive cyclization of 8-oxogeranial by iridoid synthase (ISY) followed by either a Diels-Adler reaction or a Michael addition (6). ISY was originally discovered in the Madagascar periwinkle (Catharanthus roseus, Apocynaceae), a major source of anticancer drugs derived from iridoid monoterpene indole alkaloids $(6,7)$. Interestingly, the sequence and crystal structure reported from the Madagascar periwinkle showed that ISY is not similar to TS but more closely related to the PRISE (progesterone 5 $\beta$-reductase/ISY) enzyme family, a short-chain NADPHdependent dehydrogenase $(8,9)$.

To understand the evolution of ISY in aromatic plants, a novel chemical-genomicphylogenetic approach using the transcriptomes of 48 Lamiaceae species was recently published (10). Unexpectedly, the functional validation of these candidates revealed that although ISY activates 8-oxogeranial to give an enolic intermediate it does not catalyze the consecutive cyclization into nepetalactone (11). Instead, the newly identified class of nepetalactol-related short-chain dehydrogenase enzymes (NEPS) achieves the cyclization of the reactive intermediate and controls the stereoselectivity of the outcome products (12). Furthermore, biosynthetic gene clusters composed of ISY and NEPS were identified by mining Nepeta genomes (5). Interestingly, these clusters also include major latex protein-like genes (MLPL) whose biochemical characterization showed they react in a similar manner to NEPS to form cis-trans nepetalactone stereoisomer (Fig. 1B).

With these key pieces in hand, Lichman et al. investigated the loss and re-emergence of iridoid biosynthesis during the evolution of Nepetoideae. Firstly, genome resources of Nepeta cataria and Nepeta mussinii were compared with those of the iridoid non-producer Hyssopus officinalis (indicated by a red asterisk in Fig. 1A) and confirmed that the absence of iridoids results from the loss of the ISY gene in this last species as in other Nepetoidae for which omics data are available. Surprisingly, it also directly correlates nepelactone biosynthesis to a regain of ISY in $N$. cataria and $N$. mussinii. Furthermore, phylogenetic analyses clearly indicate that ISY genes of Nepeta form a distinct clade in the Lamioideae subfamily, which strongly suggests a distinct and parallel evolution of ISYs. Secondly, the evolution of ISY in Nepeta was assessed using ancestral sequence reconstruction to infer the PRISE phylogeny. This comparative phylogenetic method was then combined with positive selection analysis and 
screening of in vitro activities of extant and predicted ancestral PRISE. Overall, the key finding of the re-emergence of ISY supports the hypothesis that an ancestral enzyme with a minor side ISY activity gradually evolved into a novel iridoid biosynthetic enzyme with high ISY activity, following gene duplication and selection (Fig. 1C).

The tour de force was to compare the evolution and diversification of NEPS with the emergence of ISY activity and to elucidate the chronology events leading to the assembly of the nepetalactone gene cluster. By comparing PRISE and NEP chronograms, the authors predicted that the gain of the most recent common ancestor of the NEPS gene was concomitant to the second gene duplication of the ISY ancestor (around $25 \mathrm{Ma}$ ago), while the NEPS family expansion occurred at the same time that ISY relative activity dramatically increased and P5 $\beta \mathrm{R}$ activity is was lost (20 to $9 \mathrm{Ma}$ ago). Ultimately, a dispersed duplication event allowed ISY to integrate into the NEPS locus while the original copy at the PRISE locus was pseudogenized since redundant ( $9 \mathrm{Ma}$ ago, today). These discoveries strongly suggest that ISY and NEPS catalytic activities have coevolved with strong interplay between corresponding genomic regions (Fig. 1C).

The evolution of iridoids in Lamiaceae thus stands out as a fascinating example for tracing the origins of plant metabolites and the re-emergence of their biosynthesis in Nepeta. The present study provides unprecedented insights suggesting that this phenomenon relies on repeated and innovative evolution further widening our knowledge of the production of nepetalactones compared to iridoids in the rest of the mint family. The proposed chronology of enzyme selection and diversification also suggests that the formation of gene clusters may not drive metabolic innovation but rather organize following enzyme evolution under strong initial pressure. Now highlighted, this twisted evolutionary story raises new puzzling questions ranging from the role of protein-protein interactions between ISY with NEPS/MLPL during the stereoselective formation of nepetalactones, to the biotic and abiotic factors responsible for the co-evolution of ISY and NEPS. Additionally, since iridoids also occur across different insect taxa, the comparison of plant and insect iridoid synthases is a crucial entry point to address the evolutionary convergence for producing these common compounds (13). The evolution of iridoids represents a remarkable model to further study enzyme and metabolite evolution across the tree of life.

\section{References (12)}

1. Wink M. (2003) Evolution of secondary metabolites from an ecological and molecular phylogenetic perspective. Phytochemistry 64, 3-19.

2. Jirschitzka J, Schmidt GW, Reichelt Ma, Schneider B, Gershenzon J, D'Auria, JC (2012) Plant tropane alkaloid biosynthesis evolved independently in the Solanaceae and Erythroxylaceae. Proc. Natl. Acad. Sci. U.S.A. 109, 1030410309.

3. Liscombe DK, MacLeod BP, Loukanina N, Nandi OI, Facchini PJ. (2005) Evidence for the monophyletic evolution of benzylisoquinoline alkaloid biosynthesis in angiosperms. Phytochemistry 64, 1374-1393.

4. Lange BM, (2015) The Evolution of Plant Secretory Structures and Emergence of Terpenoid Chemical Diversity. Annu. Rev. Plant Biol. 66, 139-159.

5. Lichman BR, et al. (2020) The evolutionary origins of the cat attractant nepetalactone in catnip. Sci. Adv. 6, eaba0721.

6. Geu-Flores F, Sherden NH, Courdavault V, Burlat V, Glenn WS, Wu C, Nims E, Cui Y, O'Connor SE. (2012) An alternative route to cyclic terpens by reductive cyclization in iridoids synthesis. Nature 492, 138-142.

7. Kries H, Kellner F, Kamileen MO, O'Connor SE. (2017) Inverted stereocontrol of iridoid synthase in snapdragon. J. Biol. Chem. 292(35) 14659-14667.

8. Kries H, Caputi L, Stevenson CEM, Kamileen MO, Sherden NH, Geu-Flores F, Lawson DM, O'Connor SE. (2012) Structural determinants of the reductive terpene cyclization in iridoid biosynthesis. Nat. Chem. Bio. 12, 6-8.

9. Nguyen T-D, O'Connor SE. (2020) The Progesterone 5 $\beta$-Reductase/Iridoid Synthase Family: A Catalytic Reservoir for Specialized Metabolism across Land Plants. ACS Chem. Biol. In press, doi: 10.1021/acschembio.0c00220. 
10. Mint Evolutionary Genomics Consortium (2018). Phylogenomic mining of the mints reveals multiple mechanisms contributing to the evolution of chemical diversity in Lamiaceae. Mol. Plant. 11, 1084-1096.

11. Sherden NH, Lichman B, Caputi L, Zhao D, Kamileen MO, Buell CR, O'Connor SE. (2018) Identification of iridoid synthases from Nepeta species: Iridoid cyclization does not determine nepetalactone stereochemistry. Phytochemistry $145,48-56$.

12. Lichman BR, Kamileen MO, Titchiner GR, Saalbach G, Stevenson CEM, Lawson DM, O'Connor SE. (2019) Uncoupled activation and cyclization in catmint reductive terpenoid biosynthesis, Nat. Chem. Bio. 15, 71-79.

13.-Beran F, Köllner T, Gershenzon J, Tholl D. (2019) The chemical convergence between plants and insects: biosynthetic origins and functions of common secondary metabolites. New Phytologist 223, $52-67$.

14.-Godden GT, Kinser TJ, Soltis PS, Soltis DE. (2019) Phylotranscriptomic Analyses Reveal Asymmetrical Gene Duplication Dynamics and Signatures of Ancient Polyploidy in Mints. Genome Biol Evol. 11, 3393-3408

Figure caption

Figure 1. Investigating the molecular basis of the re-emergence of iridoid biosynthesis in the Nepeta lineage. (A) Detection of iridoid metabolites in Nepeta. Iridoids are widely distributed in Lamioideae but are absent in the single subfamily Nepetoideae. However, there is a noteworthy exception because iridoids are found in Nepetoideae in the Nepeta genus. The phylogenetic tree represents the current understanding of the relationships of the Lamiaceae lineages, according to (12). The red asterisk corresponds to the iridoid non-producer Hyssopus officinalis. (B) Iridoid biosynthetic pathway in Nepeta. Knowledge of nepetalactones biosynthesis in Nepeta were reported in $(3,10)$. This pathway involves geraniol synthase (GES), geraniol 8-hydroxylase (G8H), 8-hydroxygeraniol oxidoreductase (HGO), iridoid synthase (ISY). Finally, nepetalactol-related short-chain dehydrogenase enzymes (NEPS) or major latex protein-like enzyme (MLPL) achieves the cyclization of the reactive intermediate and controls the stereoselectivity of the outcome products. (C) Proposed chronology of events that have likely occurred in Nepeta for the re-emergence of nepetalactone biosynthesis. 